Hugo Plomteux

Leupen

\title{
UN PRESUNTO SLAVISMO IN FRIULANO: ZÁVE 'ROSPO'
}

O. Non mancano per l'Italia studi su influssi linguistici venuti dall'esterno, sulla penetrazione, specie lessicale, dovuta alle tribù germaniche, all'espansione galloromanza, ai contatti col mondo arabo, alla colonizzazione greca, all'occupazione spagnola. Solo in alcuni rari casi, però, è stata studiata in modo sistematico l'area di diffusione di questi prestiti. Insisterei sulla necessità di analizzare con criteri geografici l'insieme degli influssi provenienti da una stessa lingua, per poter disporre di dati precisi sull'espansione proporzionale di ognuno dei prestiti e sull'importanza relativa dei vari campi semantici a cui questi elementi lessicali appartengono. In tal modo, sapremmo ad esempio non solo quali voci genovesi siano penetrate in Corsica, ma anche fino a che punto esse si siano spinte nell'interno dell'isola e se il lessico affettivo vi abbia avuto meno fortuna di quello, poniamo, mercantile. ${ }^{1}$ Sarebbe ad esempio preziosa una minuta analisi della diffusione areale degli arabismi in Sicilia, ${ }^{2}$ come è stato tentato, sia pure con una documentazione insufficiente, per gli iberismi in Sardegna ${ }^{3}$ o per gli elementi romanzi nel Tirolo (compreso l'Alto Adige). ${ }^{4}$

1 Finora, il problema dell'influsso genovese in Corsica è stato trattato solo brevemente dal Rohlfs in un opuscolo del 1941, poi ristampato nel sul volume Estudios sobre geografía lingüística de Italia (Granada 1952) 117-162 (sull'elemento genovese p. 156-161).

2 Varrebbe la pena di mettere su carte i ricchi materiali radunati e magistralmente interpretati da Giovan Battista Pellegrini nei suoi due volumi su Gli arabismi nelle lingue neolatine con speciale riguardo all'Italia, Brescia 1972 (sugli arabismi siciliani soprattutto le p.129-332 del primo volume), completandoli con i dati dell'AIS e dell'ALI. Da una tale elaborazione cartografica verrebbe, secondo ogni probabilità, messo in rilievo in un modo più concreto il maggior grado di intensità della colonizzazione araba nel Ragusano e nel Girgentino e, soprattutto, su Pantelleria.

3 José Mondéjar: Préstamos hispánicos al Sardo. Estudio de geografía lingüística in ZRPh 86 (1970) 128-167 (le p. 154-167 contengono 27 cartine). Il lavoro è basato soltanto sui primi quattro volumi dell'AIS. Conclusioni interessanti in cui si insiste sull'utilità dell'analisi geografica per gli studi sui prestiti.

4 Elmar Schneider: Romanische Entlehnungen in den Mundarten Tirols. Ein dialektgeographischer Versuch in L. E. Schmitt (ed.): Deutsche Wort- 
Per le regioni che dispongono di un atlante linguistico con una rete abbastanza stretta di punti d'inchiesta, tali studi sistematici sono di realizzazione piuttosto facile. Grazie a G.B. Pellegrini e ai suoi collaboratori padovani e friulani, il Friuli si trova a questo riguardo in una situazione privilegiata, poichè, prima tra le regioni italiane, disporrà fra non molto di un atlante linguistico completo, con materiali precisi per più di 4000 concetti, raccolti in 144 località della regione. Già dal primo volume di questo Atlante storico - linguistico - etnografico friulano (ASLEF), apparso da poco, risulta chiaramente la sua importanza per la dialettologia romanza. ${ }^{\breve{2}}$ Poichè il Friuli da più di un millennio si trova in contatto diretto con il mondo slavo, l'ASLEF fra l'altro ci fornirà - e fornisce già - materiali preziosi sia per gli slavisti che per i romanisti, sull'influsso slavo in questa marca romanza. Lo slavista vi troverà inoltre anche dati necessari all'interpretazione più adeguata degli elementi romanzi presenti in sloveno.

Certo, non sono mancati finora degli studi su questi contatti linguistici. L'influsso lessicale slavo sul friulano era già stato studiato, ad esempio, da Hugo Schuchardt,, ${ }^{6}$ poi da K. Sttrekelj e da J. Koštiál, ${ }^{7}$ per

forschung in europäischen Bezügen, Band 3 (Giessen 1963) 443-679 (41 cartine p. 651-671). Sfortunatamente, lo Schneider ha ordinato i materiali solo secondo criteri cronologici (d'altronde molto discutibili) e non, come sarebbe stato indicato, anche secondo campi semantici. L'autore poi non trae profitto dalla diffusione dei prestiti romanzi per eventuali conclusioni.

5 Dell' ASLEF è stato pubblicato finora un primo volume di carte (forme dialettali per 633 concetti, distribuite su 130 carte e 29 tavole; Padova-Udine 1972) sotto la generale direzione di G. B. Pellegrini e con la speciale collaborazione di Giovanni Frau, Paola Benincà Ferraboschi, Daniela Piccini e Laura Vanelli. Disponibile inoltre, dello stesso Pellegrini, il volume di Introduzione all'ASLEF (ib. 1972; 261 p.), il cui ultimo capitolo (p. 219-248) illustra, mediante il conciso commento ad alcune carte, la grande ricchezza lessicale del nuovo atlante rispetto ai dati dell'AIS e dell'ALI. Questa ricchezza dei materiali dell'ASLEF risulta inoltre da una serie di articoli recenti dovuti al Pellegrini (importanti soprattutto il Saggio di carte e di commenti dell'ASLEF: Commento in collaborazione con P. Benincà Ferraboschi negli Studi linguistici friulani 1 [Udine 1969] 40-98; Le denominazioni del «taglio del fieno» nelle parlate friulane in Festschrift für Karl Finsterroalder... [Innsbruck 1971] 323-340; Die Namen des «Reibeisen» für Rüben in Friaul in Festgabe Kranzmayer [Klagenfurt 1972] 53-60; Le denominazioni friulane della C a r in a A c a u is L. negli Scritti storici in memoria di Paolo Lino Zooatto [Milano 1972] 143-152; Le denominazioni della «pigna» (strobilo) nei dialetti friulani in Festschrift $W$. Giese [Hamburg 1972] 125-147), come pure ad alcuni dei suoi alunni e collaboratori (ricordo l'illustrazione di alcune carte di concetti entomologici, dovuta a Paola Benincà Ferraboschi nel citato Saggio... p. 70-96, e il commento alla carta «Millefoglio» [Achillea Millefolium L.] pubblicato da Alberto Zamboni negli Studi di filologia romanza offerti a Silvio Pellegrini, Padova 1971, 705-722).

6 Hugo Schuchardt: Slaoo-deutsches und Slabo-italienisches (Graz 1884), soprattutto p. 72--87 per gli influssi lessicali nei dialetti romanzi dell'Italia settentrionale.

7 K. Strekelj: Zur Kenntnis der slavischen Elemente im Friaulischen Wortschatz in Archiv für slav. Phil. 12 (1890) 474-478 e Nachtrag nella stessa rivista 
essere recentemente ripreso dal Pellegrini. ${ }^{8}$ I materiali pubblicati nel' ASLEF ci permetteranno però di tener conto dell'area di diffusione di ognuno degli slavismi e della relativa importanza di questi nell'insieme del lessico friulano. $E$ ' perciò sperabile di avere in un futuro non troppo lontano, un lavoro di lessicologia friulana comparabile al recente Slavoteutonica di Gïnter Bellmann, lavoro che è dedicato all'influsso slavo in un'area dialettale della Germania confinante con zone di lingua slava. ${ }^{9}$ In un tale studio, non solo verrebbero per la prima volta messi in rilievo casi finora sconosciuti di influssi slavi nel Friuli, ma occorrerà inoltre riesaminare minuziosamente le etimologie slave già proposte. Gli appunti che seguono, vogliono contribuire modestamente a questo controllo critico.

1. Fra i presunti slavismi del friulano che non resistono ad un'analisi attenta, bisogna menzionare záve «rospo»,10 considerato ancora spesso, e a torto, come un prestito dallo sloveno žaba «rana».11

Credo sia utile ritornare ancora una volta sull'origine della voce friulana per ripetere, dettagliatamente, perchè tale etimologia sia da scartare, tanto più che altri tentativi etimologici, meno appariscenti certo, ma più attendibili, continuano ad essere ignorati od almeno passati sotto silenzio dagli specialisti del friulano.

31 (1910) 203-209; J. Koštiál: Slavische Lehnwörter im Friaulischen. Nachträge zu Prof. Strekeljs Abhandlungen ib. 34 (1913) 292-298.

${ }^{8}$ G. B. Pellegrini: Contatti linguistici slavo-romanzi (dispense universitarie ciclostilate, Padova 1969) 168-172 e 197-201. Alcuni elementi ne sono stati ripresi anche nell'Introduzione all'ASLEF (Padova 1972) dello stesso autore, p. 78-79 e 86-87. Si veda anche l'elenco fornito da G. Marchetti nei suoi Lineamenti di grammatica friulana ${ }^{2}$ (Udine 1967) 41-44.

- Giunter Bellmann: Slaboteutonica. Lexikalische Untersuchungen zum slamisch-deutschen Sprachkontakt im Ostmitteldeutschen (= Studia Linguistica Germanica 4), Berlin-New York 1971, XII + 356 p.; 65 cartine nel testo. Il lavoro contiene un ricco capitolo introduttivo su Sprachentricklungen unter den Bedingungen des Sprachkontaktes (p. 9-61), della massima importanza generale.

${ }_{10}$ Quanto alla trascrizione fonetica, è da tenere presente che uso $z$ e $\check{Z}$ per le sonore corrispondenti a $s$ e š, e che l'accento tonico è indicato con ' $o$ ' sopra alla vocale. Una forma tipizzata, cioè citata facendo astrazione dalle sue varianti fonetiche, viene messa sotto $i$ segni $r$...?.

11 Così ad esempio già un certo Giuseppe Domenico Della Bona nel $\mathrm{Ca}$ lendario per l'anno 1849 , pubblicato dalla società agraria di Gorizia.... p. 78 (cit. da K. Strekelj nell'Archio für slavische Philologie 31 [1910] 203), poi il Pirona nella prima edizione del suo Vocabolario friulano (Venezia 1871, p. XCII), il Gartner (Rätoromanische Grammatik; Heilbronn 1883, 32), Gustav Meyer (Etym. Worterbuch der albanesischen Sprache, Strassburg 1891, 399), Meyer-Lübke (REW 7593) e, più recentemente, G. B. Pellegrini (Contributi... cit., p. 199). Anche il Marchetti, che in un plimo tempo aveva pensato per questo termine ad un'origine prelatina o preceltica (Ce Fastu 9 [1933] 130), ha poi optato per l'etimologia dallo slavo (Lineamenti... cit., p. 43). Molto cauto il Prati, che segnalò la nostra voce friulana come un termine «che s'è forse risentito dallo slov. žaba» (AGI 18 [1914-1922] 445). 
2. E' ovvio che, a prima vista, l'origine slava si záne sembra corroborata da vari argomenti. Così, in favore della derivazione dallo sloveno, si potrebbe invocare, oltre alla somiglianza fonetica, il fatto che $i$ forestierismi non mancano fra le denominazioni romanze del rospo o della rana (2.2), che žaba è molto diffuso in territori confinanti ma non appartenenti al dominio slavo (2.3), e che un'oscillazione fra $i$ termini in uso per la rana e quelli abitualmente adoperati per il rospo non può essere un ostacolo a questa etimologia, dato che tali scambi sono anch'essi molto frequenti (2.1). Illustriamo prima brevemente questi punti.

2.1 Il fatto che žaba significa «rana», mentre in friulano il tipo ${ }^{\ulcorner} z a ́ b e^{\urcorner}$ è solo in uso per il rospo, ${ }^{12}$ non sarebbe di per sè un argomento per mettere in dubbio un'eventuale filiazione diretta fra i due termini. Infatti, un tale slittamento semantico non solo è molto semplice, ma è inoltre frequente anche in altre lingue. Oscillazioni di uno stesso tipo lessicale fra i significati «rana» e «rospo» sono ad esempio documentati per il tedesco, per l'ucraino, ${ }^{13}$ per certi dialetti dell'Olanda del NordEst, ${ }^{14}$ e anche per certi dialetti italiani meridionali dove rana appare a volte nel significato di «rospo ${ }^{15}$ e nel Friuli stesso, dove, per designare la rana, si usa su una vasta zona il tipo ${ }^{\ulcorner}{ }_{k r o ̀ t}{ }^{\urcorner},{ }^{\ulcorner} k_{r o ̀ t a}{ }^{\top}$ che risale al tedesco Kröte «rospo».16

12 Per il Friuli, mi fondo sui materiali, ancora inediti, dell'ASLEF, che ho potuto consultare all'Istituto di Glottologia dell'Università di Padova grazie alla gentile concessione del Prof. G. B. Pellegrini, che qui ringrazio sentitamente. In seguito mi riferisco anche, oltre che alle carte dell'AIS, ai dati inediti dell'ALI, consultati a Torino nel 1961--62, grazie ai direttori dell'ALI, di cui ricordo soprattutto il compianto maestro Benvenuto Terracini. Solo

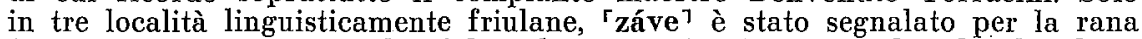
(ma ivi sempre accanto ad un'altra denominazione); in tre altre località lo si adopera per la raganella. Per il rospo, invece, il tipo ‘zábe è è vivo nella quasi totalità dei comuni friulani.

${ }_{13}$ Cfr. Günter Bellmann: Slaooteutonica... cit., p. 130, con esempi per i dialetti tedeschi nei pressi di Breslau/Wroclaw e con rinvii ad una tesi dattiloscritta di Marburgo di Ursula Wiepen (1965; esempi tedeschi, norvegesi antici, anglosassoni), ad un articolo di Helmut Klaus (Zur Namengemeinschaft bei 'Frosch' und 'Kröte' in Zeitschr. für Mundartforschung 25 [1957] 39-56) e al Russisches etym. Wörterbuch 1 (Heidelberg 1953) 407 di Max Vasmer, dove però le oscillazioni all'interno del mondo slavo sono praticamente neglette (lo sono del tutto nella recente edizione russa dello stesso dizionario, Etimologičeskij Slooar russkogo jazyka 2 [Moskva 1967] 31)

${ }_{14} \mathrm{~K}$. Heeroma: Taalatlas ban Oost-Nederland en aangrenzende gebieden, 2 e aflevering + Toelichtingsboek (Assen 1960): cfr. la carta no 16 e, nel volume di commento, la p. 95.

15 Cfr. AIS III 455.

${ }_{18}$ Cfr. AIS III 453, ALI domande 4702 e 4705, ASLEF domande 185 e 187-189. Nelle oasi alloglotte germaniche d'Italia (Val d'Aosta, Sette Comuni vicentini; Tredici Comuni vicino a Verona) e nell'Alto Adige, e nelle zone influenzate da questi centri tedeschi, kröte sopravvive nel significato originario di «rospo» (cfr., oltre agli atlanti citati, anche $i$ materiali raccolti dal Garbini: Antroponimie ed Omonimie nel campo della zoologia popolare ... 1 [Verona 1925] 279; Giuseppe Cappelletti nell'Italia dialettale 12 [1936] 184 per 
Una simile evoluzione semantica si sarebbe dunque potuta produrre facilmente in Friuli anche nel caso dello sloveno žaba.

2.2 Benchè non sia direttamente chiaro per quale motivo, ${ }^{17}$ è un fatto che in parecchie zone dell'Europa ci sono dei prestiti linguistici fra le denominazioni del rospo o della rana. Anche sotto questo aspetto, un eventuale passaggio di una parola slava in un'area contigua romanza non desterebbe alcuna meraviglia. Limitandoci ai dialetti romanzi d'Italia, abbiamo già menzionato la penetrazione del tedesco Kröte in praticamente tutto il Friuli, con cambiamento di significato («rana» invece di «rospo»). In Sicilia, e per influsso siciliano anche in alcune località della provincia di Catanzaro, la rana verde (rana viridis) e anche la raganella (hyla arborea) portano tuttora una denominazione di origine araba: ${ }^{\top} \breve{g} u r a ́ n a{ }^{7},{ }^{\top}$ čurána ${ }^{7}$, della quale avremo da riparlare alla fine di questo articolo. ${ }^{18}$

2.3 Ricordiamo ancora, come ultimo fattore in favore di una ipotetica derivazione del friulano ${ }^{\Gamma} z a ́ b e^{\top}$ dallo sloveno, che žaba ha avuto

i Tredici Comuni; G. S. Martini: Vocabolarietto gardenese-italiano [Firenze 1953] sotto la voce; Angelico Prati: Etimologie venete. [Venezia-Roma 1968] 52 per il vicentino e il triestino; ecc.). Krota «rospo» anche in certi dialetti sloveni (Wolf-Pleteršnik Deutsch-slovenisches Wörterbuch 1 (Ljubljana/Laibach 1860) 906; l' ALI domanda 4707 documenta ' kruóta $^{\top}$ anche per Divača/Divaccia, Novigrad/Cittanova d'Istria e Ilir. Bistrica/Villa del Nevoso. Non so fino a che punto questi materiali istriani diano indicazioni sul parlare della popolazione autoctona del periodo prebellico. Per gli influssi etnici e linguistici degli insediamenti tedeschi in Istria rimane tuttora utile Sextil Puşcariu: Studii istroromâne 2 (Bucureşti 1926) 29-36 (soprattutto p. 31 ; con bibliografia).

17 Fra i motivi eventuali annovererei il tabù, poichè secondo molte tradizioni popolari il rospo e la rana sono considerati come animali magici o comunque temibili. Rinvio in proposito ad un mio breve saggio su Le crapaud: magie et maléfice pubblicato nella Revue de linguistique romane 29 (1965) 132-140. Altrove è stato sottolineato che, in caso di tabù̀, si fa spesso ricorso ad un prestito da una lingua straniera: cfr. molti esempi apud W. Havers, Neuere Literatur zum Sprachtabu (Wien 1946) 35, 39, 48, 49, 51, 52, 67, 94, 101, 111,135 e soprattutto $128-132$. In alcuni casi il prestito di denominazioni estere per il rospo o per la rana può avere avuto anche, all'inizio, un semplice conno tato espressivo, in tal senso che un prestito da una lingua straniera prende facilmente un valore peggiorativo. Vari esempi ed una ricca bibliografia di questo fenomeno, in Slavoteutonica cit. di G. Bellmann, p. 39-40. Per i contatti slavo-romanzi, si vedano anche i saggi di Ivan Popovic: Quelques termes péjoratifs serbo-croates d'origine romane in: Vox Romanica 16 (1957) 219-223 e di Žarko Muljačić: Dalmatske Studije I: casalis $>$ cosel $>* \operatorname{cosal}>k o s(a) o$, ala in: Sveučilište u Zagrebu. Filozofski Fakultet Zadar. Radovi 1 (1959-1960) $85-100$.

${ }^{18}$ L'arabismo (< arabo magrebino ğarân) copre praticamente tutta l'isola, eccetto una striscia lungo la costa orientale (Messina, Catania, Siracusa): si vedano i materiali dell'AIS III 453-454, ALI domande 4702 e 4706, Garbini op. cit. 1. 843-845, Rohlfs Dizionario dialettale delle Tre Calabrie 1 (Halle e Milano 1932) 184 e 212-216. Per l'etimologia si possono consultare P. Gabrielle Maria da Aleppo: Le fonti arabiche del dialetto siciliano 1 (Roma 1910) 212-213 e ora soprattutto G. B. Pellegrini: Gli Arabismi... cit., 1 (Brescia 1972) 200. 
molta fortuna anche in territori non slavi. Non solo questo slavismo è penetrato in certi dialetti tedeschi di confine, ${ }^{19}$ ma lo si trova anche come prestito in vari dialetti albanesi (aree di Gjakovë/Đakovica, EIbasan, Shkodra/Scutari, Vlora/Valona), ${ }^{20}$ in qualche dialetto macedorumeno, ${ }^{21} \mathrm{e}$ in parecchi dialetti neoellenici ${ }^{22}$

3. Nonostante tutti questi elementi, che sembrano appoggiare l'evidenza dell'etimologia slava della voce friulana, ci urtiamo a due difficoltà finora non abbastanza messe in rilievo: una di ordine fonetico

${ }^{19}$ Günter Bellmann: Slaooteutonica... cit. p. 116 (cartina) e 130-131 sulla diffusione di ${ }^{r} z \bar{a} b \partial^{7}$ nei dialetti tedeschi vicino a Breslau/Wrockaw (periodo fra le due guerre mondiali).

${ }^{20}$ Gustav Meyer: Etymologisches Wörterbuch der albanesischen Sprache (Strassburg 1891) 399 e soprattutto Stuart E. Mann: An Historical Albanian-English Dictionary (London 1948) 585, sotto zhabë «kind of frog»; žab, žaba «rana», «raganella» anche nel dialetto albanese di Borgo Erizzo/Arbanasi presso Zadar, secondo Carlo Tagliavini: L'albanese di Dalmazia (Firenze 1937) 296.

${ }^{21} \mathrm{~T}$. Papahagi: Dicționarul dialectului aromîn general gi etimologic $(\mathrm{Bu}-$ curești 1963) 590, che riprende jabă «rospo» dall'ottocentesco Dictionar macedo-romîn di Ioan Murnu, rimasto inedito.

${ }^{22}$ L'area di diffusione non risulta chiaramente dalle fonti che ho qui a mia disposizione. Lo slavismo manca nei dizionari comuni, (anche in quello di

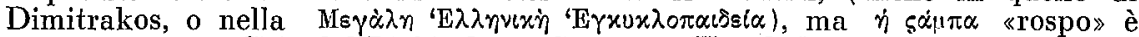
stato notato per Chio dal P. Girolamo Germano (Vocabolario italiano e greco .... Roma 1622, p. 265; cfr. H. Pernot: Etudes de linguistique néo-hellénique. III. Textes et lexicologie des parlers de Chio, Paris 1946, p. 404 e, per il valore del vocabolario del p. Germano: H. Pernot: Phonétique des parlers de Chio, Fontenay-sous-Bois / Paris 1907, p.4), poi nel Tesoro della lingua greca-bolgare ed italiana, (Paris 1709) del padre Alessio da Somavera. Tramite il Germano e il Somavera, la voce è passata anche in alcuni dizionari compilati nel primo Ottocento (ad es. Teutsch-Neugriechisches Wörterbuch di Karl Weigel, Leipzig,

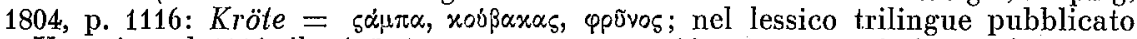

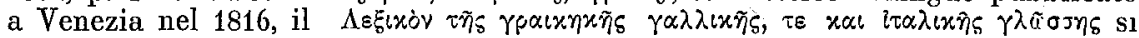

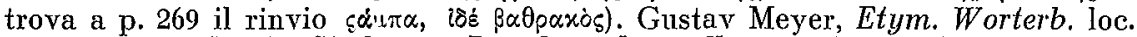
cit. e Neugriechische Studien 2: Die slavischen, albanesischen und rumänischen Lehnmörter im neugriechischen (Wien 1894), p. 27, menziona inoltre il greco

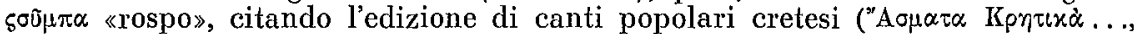
Leipzig 1876) di A. Jannarakis. Lo slavismo sarebbe dunque arrivato fin sulle isole? - Basandomi su dati inediti del lessico storico del neogreco (Atene) fornitimi gentilmente dal. collega Nicolaos Kontosopoulos, posso ora precisare che, anche se l'indicazione dello Jannarakis non si riferisce in questo caso ad un uso lessicale cretese, è comunque accertato che lo slavismo è profondamente penetrato non solo nel Nord della Grecia (Epiro, Macedonia, Tracia) ma fino in Laconia, nell'eparchia di Olimpia, di Gortinia, ecc. Accanto ad altre indicazioni, che qui per brevità devo tralasciare, Kontosopoulos mi ricorda che altri zoonimi slavi hanno avuto una larga diffusione nei dialetti

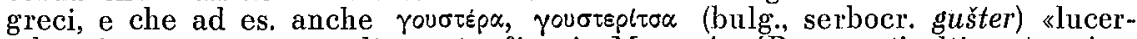
tola» si trova su area molto vasta, fino in Messenia. [Per quest' ultimo termine, la cui espansione al difuori dell' ambito slavo offre qualche paralellismo con quella di žaba, si vedano anche G. Meyer: Neugriechische Studien 2, cit., p. 24; G. Rohlfs in Mélanges... Petrovici, Cluj 1958, 411; A. Cioranescu: Diccionario etimológico rumano, Tenerife $1966,386=\mathrm{n} .3935$.] 
(3.2), e soprattutto una concernente l'area di diffusione di questo pre. sunto slavismo (3.3).

3.1 Si nota già subito controllando i materiali dell'ASLEF per «rospo» e simili, che fra area slovena e area friulana non c'è continuità di terminologia, al difuori del caso problematico di žaba/ $z a ́ b e^{\top}$.

L'ASLEF contiene materiali per ben nove località slovene ai limiti orientali del Friuli. ${ }^{23}$ Per «rospo» ecc., le forme dialettali ivi raccolte sono le seguenti: ${ }^{24}$

punto ASLEF

6 a Pontebba-Laglesie

7 a Ugovizza

34 a Oseacco

34 b Stolvizza

46 a Pradielis-Lusevera

67, a Cergneu di Sopra «1ospo»

kî́ta

pastrníena króta strníčenza

kròća

škốrna žába

žába

žába

88 a S. Pietro al Natisone źába Vernasso

219 Sgonico «rana» «raganella»

žába

rékuz

žába

žába

kròta

króta te

zenéna

žába/krót krótza/króta

zeléna žába žába gráćule

Come si poteva prevedere, la terminologia è praticamente tutta slovena: oltre a žaba (žabica, zelena žaba), ${ }^{2 \check{5}}$ krota (krotica), rega (qui il diminutivo al punto 7 a), ${ }^{26}$ e un termine più raro, quale strniščnica (qui forma alterata allo stesso punto 7 a), ${ }^{27}$ troviamo un tipo finora non

${ }^{23} \mathrm{Su}$ queste località, ormai quasi tutte diventate bilingui o trilingui, si consulti il ricco capitolo di G. B. Pellegrini: Introduzione all' $A S L E F$ cit., p. 53-91, nonchè l'articolo dello stesso autore pubblicato negli Studi linguistici in onor'e di Vittore Pisani, Brescia 1969, 761-776.

${ }^{24}$ Non dispongo per ora dei dati per il punto 70 dell'ASLEF (Savogna; inchiesta di Ugo Pellis).

${ }_{25}$ Per l'articolo (in króta te zenéna) e la sua diffusione in alcuni dialetti sloveni del Friuli, cfr. G. B. Pellegrini: Introduzione all' $A S L E F$... cit., p. 87-88 (e già, negli scritti anteriori dello stesso autore: Contributi... cit., p. 204-205 e, con rinvii bibliografici, nell'articolo Contatti linguistici slaoo-friulani in Studi linguistici in onore di Vittore Pisani, Brescia 1969, p. 772-773).

${ }^{26} \mathrm{Cfr}$. sloveno rega «rana verde».

27 Pleteršnik: Slonensko-nemški slooar 2 (Ljubljana 1895) 952 cita strniščnica «(1) was unter das Getreide gesäet wird und auf dem Stoppelfeld wächst, z. B. die Stoppelrübe, der Stoppelklee; (2) [Krajn, Kranj. Gora] die gemeine Kröte», denominazione da comparare con Kleefeldtatschka «rospo» in un dialetto tedesco (W. Mitzka Deutscher Wortatlas IV [Giessen 1955] 27. Comp. anche, a Predielis - Lusevera (ASLEF punto 46 a) fartáiariza «ululone rosso» (bombinator igneus $=$ ASLEF domanda 189$)=$ sloveno ortarica «Gärtnerin» (Pleteršnik, op. cit. 2, p. 800)? 
segnalato per lo sloveno, pastrníena krota, anch'esso indubbiamente sloveno, ${ }^{28}$ e l'interessante škốrna žaba, ${ }^{29}$ tipo da comparare con il friulano $\left\ulcorner\right.$ krot malós ${ }^{\top}$ ecc. $^{30}$

L'unica denominazione non slava fra le forme registrate in queste località slovene è il tipo ' ${ }^{\top}$ grácule ${ }^{\top}$, abbastanza comune anche altrove in Friuli e di origine apparentemente onomatopeica. ${ }^{31}$

3.2 Di tutte queste denominazioni slovene, non c'è più traccia nella confinante zona friulana. Solo žaba sembrerebbe continuare in area romanza, ma in veste romanizzata. ${ }^{32}$ Sembrerebbe: infatti, già per ragioni fonetiche questa forma friulana ${ }^{\top} z^{\prime} a e^{\top}$ potrebbe difficilmente provenire dallo slavo, poichè la lenizione di $-b$ - intervocalica verosimilmente era del tutto finita quando, nel sesto secolo, gli Slavi entrarono in diretto contatto con le popolazioni romanze del Friuli. ${ }^{33}$

Non è qui il luogo per riprendere fin nei particolari il problema dell'indebolimento di una consonante semplice posta fra vocali, fenomeno comune in molte lingue indoeuropee ${ }^{34}$ (e poco ci importa qua se anche nella Romània la si debba interpretare come una tendenza dello sviluppo interno di varie parlate romanze, piuttosto che come tratto tipico di un malsicuro sostrato celtico). ${ }^{35}$ Comunque sia, è noto che le sonore labiali

${ }_{28}$ Da pastir «pastore»? Spero di riprendere in altra sede le varie denominazioni europee dei batraci anuri, derivanti dalla credenza popolare secondo cui il rospo (come le bisce, la salamandra, il caprimulgo e così via) succhierebbero il latte del bestiame. Il tipo 'pastore' appare spesso in tali denominazioni.

29 Pleteršnik, op. cit. 2, p. 499: skrn, skrna «verdorben» (Kranj Gora, ecc.).

30 Fra le denominazioni friulane della raganella, l'ASLEF (dom. 187) dà krót malín per Nimis, punto 67; per l'ululone rosso (dom. 189) krot malós a Moruzzo (punto 100), krote maloze a Colloredo-Mels (punto 80 a). Per l'etimologia del termine 'malós' stesso, che in friulano esiste solo con riferimento al rospo, comp. FEW VI, 1, 88 a-b e soprattutto $124 \mathrm{~b}, 125$ b e 127 a.

31 Esclusa una derivazione da grassus, come aveva proposto Meyer-Liibke (REW 2299); cfr. piuttosto FEW II 1295 a, sotto krats. - Da ricordare che in Friuli questo stesso tipo lessicale è frequente anche nel significato «raganella per la settimana santa» (AIS IV 789).

32 Solo ad Attimis-Racchiuso e Ronchi dei Legionari - Cave di Selz (ASLEF punti 68 a e 199 a), villaggi friulani sul bordo dell'area slovena, il rospo porta un nome prettamente slavo: žába.

33 Per la cronologia degli insediamenti sloveni in Friuli, basti rinviare alle indicazioni di sintesi fornite di recente da G. B. Pellegrini: Contatti linguistici... (cit.) 166-168, e la pur citata Introduzione all'ASLEF, soprattutto p. $74-77$.

34 Rinvio ad esempio a M. Grammont: Traité de phonétique (Paris 1933; varie ristampe anastatiche) 200 ed ai lavori di C. Battisti: Le dentali esplosive intervocaliche nei dialetti italiani (Halle 1912) 49-54 e soprattutto Fonetica generale (Milano 1938) 309-321.

${ }_{35}$ Sulla lenizione celtica informa fra l'altro Martinet Economie des changements phonétiques (Bern 1955) 257-296. La teoria del sostrato nel caso della lenizione, ampiamente illustrata (ma non condivisa) nel lavoro recente di Fr. H. Jungemann, La teoría del sustrato y los dialectos hispano-romances y gascones (Madrid 1955), 132-152, viene criticata soprattutto dagli ispanisti quali Malmberg (ad esempio Occlusion et spirance dans le système consonantique 
$-b$ - e $-b$ - cominciano a confondersi già nelle epigrafi latine del periodo repubblicano ${ }^{36}$ e che d'altra parte quest'evoluzione doveva essere finita alla fine dell'Impero, cioè quando gli Slavi entrano in contatto con la popolazione romanza del Friuli. Fra gli slavismi in friulano, non conosco nessun esempio che abbia ancora partecipato alla lenizione.

3.3 Anche se l'argomento fonetico non dovesse essere decisivo nel caso di $\left.{ }^{\ulcorner} z a ́ v e\right\urcorner$, non disponendo di studi rigorosi sui limiti cronologici delle evoluzioni fonetiche, anche l'area di diffusione del presunto slavismo friulano è un ostacolo per ammettere una filiazione diretta dello sloveno.

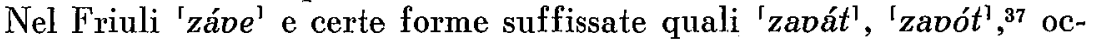
cupano un'area continua ad est del Tagliamento (salvo nel Nord). Ad ovest di questa area compatta si trovano i punti friulani al dilà del fiume dove il nostro tipo è usuale (punti 11-15). Appaiono inoltre dieci località del Cadore e del Trentino (punti 1-10), dove il rospo porta delle denominazioni ovviamente collegate con quelle friulane. Si confrontino:

a) nei punti friulani:

11 Claut (ASLEF 40)

12 Vico (ASLEF 22 a)

13 Forni di Sotto (ASLEF 23)

14 Barcis (ASLEF 54)

15 Grizzo (ASLEF 73 a)

b) nei punti dolomitici:

1 Tuenno (= AIS 322)

2 Faver (=AIS 322)

3 Trento (= ALI 238) zavát

źavát

źaf

zovát; zágu

źovát

çavát

ćavát

oavát «rospo»

《rospo»

«rospo»

《rospo»

«ululone rosso»

《rospo»

«rospo»

《rospo ${ }^{38}$

de l'espagnol in Mélanges... K. Michä̈lsson, Göteborg 1952, 356-365 e La structure phonétique de quelques langues romanes in Orbis 11 [1962] 164-169) e Alarcos Llorach: Fonología española (Madrid 1964) 244--254. Anche Weinrich cerca di inserire la lenizione nella storia interna delle lingue romanze occidentali (Phonologische Studien zur romanischen Sprachgeschichte, Münster 1968, 105143 passim; il capitolo sulla sonorizzazione è stato commentato da Kurt Baldinger in ZRPh 74 [1958] 456-459). Un'esposizione cauta delle varie ipotesi sulle cause della lenizione è stata data anche dal latinista svedese Bengt Löfstedt: Studien über die Sprache der longobardischen Gesetze, Uppsala 1961, 139-142.

${ }_{36}$ Molti casi del conguaglio fra $-b$ - e - $b$ - in latino sono stati riuniti.e discussi dal Battisti: Avviamento allo studio del latino volgare (Bari 1949) 153-156. Utili anche le indicazioni di Elise Richter: Beiträge zur Geschichte der Romanismen 1 (Halle 1934) 60-62 e di Maria Bonioli: La pronuncia del latino nelle scuole dall'Antichità al Rinascimento 1 (Torino 1962) 92-94.

${ }_{37}$ In friulano, il suffisso -at, unito a nomi di animali, esprime sia «una semplice variante del primitivo, anzi talora un equivalente, nel quale caso il suffísso è pleonastico», sia «il nome dell'animale maschio, derivandolo da un nome di animale di forma femminile» (Giorgio De Leidi: $I$ suffissi nel friulano, tesi di laurea inedita dell'Università di Padova 1945-1946, p. 82-85). In friulano è abbastanza comune anche raspát «rospo» ecc. (cfr. Il Nuovo Pirona... cit., p. 852 e i materiali dell'ASLEF domande 188 e 189, passim).

${ }_{38}$ Varietà molto grande. 
4 Rovereto $^{3 \theta}$

5 Viarago (= AIS 333)

6 Roncegno (=AIS 344)

7 Caldonazzo (= ALI 239)

8 Predazzo (= AIS 323)

9 Canal San Bovo (= ALI 233)

10 Vodo Cadore (= ALI 226) čaváz

čavatón

savatún

oavatóm

ćavatón

@avát

čavát «rospo»

«rospo»

«rospo $\rangle^{38}$

«rospo $\rangle^{38}$

«rospo ${ }^{38}$

«rospo ${ }^{38}$

«rospo»

Nessuno ha mai pensato di interpretare queste forme come slavismi, e a buon diritto. Troviamo inoltre alcune forme simili anche verso la pianura:

\section{Valrovina (= ALI 248) \\ 17 Villabartolomea Legnago ${ }^{40}$}

@avatón

sava, saba, sabòto «rana fusca»

«rospo»

Non c’è nessun motivo per staccare queste forme da quelle friulane, o da quelle dolomitiche, come non c'è nessun motivo per distoglierne le forme provenzali, quali: sabatas, sabatà, sobotà, o savà nelle HautesAlpes, sabô nel dipartimento Rhône, échaoô ad Étampes (dip. Seine et Oise), o nei dialetti pirenaici sapaou, sapou, sabaou, sobaou sul versante gallo-romanzo, apho, afo, sapho, sapo nei dialetti baschi, sapo in spagnolo e portoghese, ecc. ${ }^{41}$

In questi ed altri casi similita non può ovviamente trattarsi di slavismi, come non può essere invocata una fortuita somiglianza fra alcune di queste forme e il termine ' ciabatta?.43 Improbabile anche

30 Cfr. Prati AGI 18 (1914-1922) 444; per Sacco-Rovereto, i materiali dell'ALI (punto 245) danno oavatóm per una varietà particolarmente grande di rospo.

40 Garbini: Antroponimie... cit., p. 280. - Per le forme con $-b$ - si potrebbe pensare ad un incrocio con botta «rospo»> sabòto donde in un secondo tempo la retroformazione saba. Un'altra soluzione è indicata nel FEW XI 217 a.

${ }^{41}$ Per le varianti e l'esatta localizzazione, si vedano soprattutto E. Rolland: Faune populaire de France 3 (Paris 1881), p. 47, e 11 (Paris 1910), p. 87 e 123; Sainéan: La création métaphorique .... le chien et le porc... (Beiheft ZRPh 10; Halle 1907) 115; ALF carta 346; Atlas ling. de la Gascogne 1, carta 40; Corominas DELC IV 145 a e 146 b; FEW XI 217 a; per il basco si consulti, oltre a $\mathrm{M}$. Löpelmann: Etym. Wörterb. der baskischen Sprache 1 (Berlin 1968) 66-67, anche la Fonética histórica pasca (San Sebastián 1961), p. 291-292 di Luis Michelena a proposito dell'alterazione, regolare in basco, fra varianti con o senza sibilante prevocalica iniziale.

${ }^{42}$ Comp. nelle Valli Giudicarie sap «rospo» a Roncone TN, tšap «id.» a Tiarno di Sotto TN (AIS carta 455, punti 340 e 341). Il rapporto fra tutte queste voci e l'area di šat ecc. «rospo» nell'Engadina, nella Lombardia e nel Piemonte nord-orientale (cfr. AIS carta cit.) non è ancora chiaro (ma sembra utile prendere in considerazione una variante *SATTUS accanto a "SAPPUS, *SAPUS, come ha fatto Tagliavini: Nuovi contributi ... . Comèlico, Venezia 1944, 131-132).

43 Anche un confronto fra le forme friulane raccolte per la domanda «ciabatta» dell'ALI (dom. 6339) e quelle del tipo zaoát «rospo» dimostra chiaramente che, in Friuli, le denominazioni del rospo non possono essere derivate dal 
che si tratti di un'onomatopea. ${ }^{44}$ Poichè è esclusa inoltre un'etimologia dal fondo latino, anche gli specialisti del friulano dovranno riconnettere il loro termine al tema prelatino *SAP(P)US già proposto dallo Jud sessant'anni or sono, e ormai largamente ammesso dai romanisti. ${ }^{45}$

4. Sarebbe dunque completamente fortuita la coincidenza di due aree contigue, quella slovena di žaba e quella friulana di záve non apparentate fra di loro? Geneticamente si tratta di due vocaboli non collegati. E' però probabile che la vicinanza di žaba abbia permesso al termine friulano, così simile, di mantenersi più facilmente, com'è sicuro che per il fonetismo della consonante iniziale, le forme friulane si risentono spesso di quella slava. Qualcosa di simile è d'altronde anche avvenuto nel caso delle denominazioni siciliane dei batraci, dove i continatori dell'arabo ğarán si sono verosimilmente mantenuti ed estesi grazie alla somiglianza con i continuatori di rana. In certi dialetti siciliani, si può parlare di un incrocio vero e proprio, così ad esempio nella colonia gallo-italiana di Sperlinga dove in ćuráina «rana» si riconosce ancora facilmente ráina «rana» in uso in certi dialetti liguri marginali. ${ }^{46}$

termine locale per la ciabatta. In altre regioni, qualche caso isolato di ravvicinamento fonetico fra le denominazioni del rospo e quelle della ciabatta sarà un fatto seriore, dovuto ad etimologia popolare (penso al trentino čavát, zavát in uso sia per il rospo che per "villanzone, tanghero» $\mathrm{e}$ ad alcuni casi simili, segnalati dal Prati AGI 18 [1914-1922] 444-445). Isolato è anche il tipo skarpáoa e derivati, segnalato per il rospo nella Valsugana e a Pozzoleone VC (Prati, loc. cit. e Diz. oalsuganotto, Venezia-Roma 1960, 157: ALI domanda 4707 punti 240 e 259) cioé in mezzo all'area čavát ecc. indicata sulla nostra cartion, e perciò da interpretare come un altro esempio di rifacimento seriore, tanto più che in tutta l'Europa non conosco nessun altro caso di una denominazione di batraci derivata da un termine designante un tipo di calzatura o un indumento qualsiasi.

44 Solo Corominas DELC IV 145 b (e così anche nel Brebe dicc. etim. de la lengua castellana, Madrid 1961, 511) non esclude che si possa trattare di una «formación onomatopeica muy antiga, que imitaría el ruído del animal al caer de vientre en tierra mojida» (Brebe dicc.), almeno per le voci iberiche qui ricordate.

45 Oltre al Jud (Dalla storia delle parole lombardo-alpine in Bulletin de dialectologie romane 3 [1911] 11, in favore di un $* \mathrm{SAPA}$ ), un tema prelatino è stato accettato (spesso con riferimento esplicito anche alle forme friulane) ad esempio dal Meyer-Lübke (REW 7593 *SAPPUS; solo per le forme iberoromanze), da von Wartburg (FEW XI 217 a "SAPPUS/*SAPUS), da J. Hubschmid (Sardische Studien, Bern 1955, 117-118; Enciclopedia lingüística hispánica 1, Madrid 1960, 47), dal Corominas (DELC IV 145 b: secondo il Corominas, un tema prelatino potrebbe forse essere preso in considerazione ma solo per le forme iberoromanze). Nell'ambito dei «friulanisti» invece, questa etimologia è stata passata sotto silenzio.

${ }_{40}$ Il termine di origine araba (cfr. qui sopra, nota 18) di Sperlinga, è preso dall'AIS, carta 454, punto 836; per il tipo ráina «rana» e la sua area di diffusione nella Liguria e zone limitrofe, rinvio al mio Lessico dei dialetti della Liguria orientale: la Val Graveglia, Bologna 1974, sotto ráña. 
La differenza fra il caso siciliano e quello friulano è però che in Sicilia il prestito arabo subisce l'influsso del termine indigeno che lo sorregge in parte, mentre in Friuli è il termine indigeno che, grazie ad una somiglianza formale e semantica con un termine sloveno, ne trae forse maggiore vitalità, e ne subisce un lieve influsso fonetico, pur rimanendo ancora ben chiara la sua fisionomia non slava.

\section{Poozetek}

\section{$\check{Z} A B A$ : DOMNEVNA IZPOSOJENKA IZ SLOVENSČINE V FURLANŠČINO}

Slovansko-romanski medsebojni jezikovni vplivi so zlasti vidni v stičnih pokrajinah in to je vzrok, da so furlanski vplivi v slovenščini in slovenski $v$ furlanščini pritegnili zanimanje pomembnil jezikoslovcev: dovolj je omeniti Huga Schuchardta in za njim Štreklja in Koštiala ter, bliže našemu času, Pellegrinija. Avtor si je zadal nalogo, da preveri, ali je mogoče imeti furlansko besedo zave za delno prilagojeno izposojenko iz slovenske besede žaba, kot to misli večina furlanskih etimologov in kakor navaja tudi Pirona v svojem furlanskem besednjaku. Avtor misli, da govori proti taki razlagi lenizacija $b>0$ $\mathrm{v}$ zave in pa tudi razprostranjenost izraza daleč preko ozemlja, ki meji na Slovenijo in je torej potencialno izpostavljeno tujemu jezikovnemu vplivu. Predlaga kot najverjetnejšo etimologijo predlatinski *SAPPUS, na kar je pomislil na začetku našega stoletja že švicarski romanist Jud. 